\title{
Self-Checking Combinational Circuits with Unidirectionally Independent Outputs
}

\author{
A. MOROSOW ${ }^{a}, *$, V. V. SAPOSHNIKOV ${ }^{b}$, VL. V. SAPOSHNIKOV ${ }^{b}$ and M. GOESSEL ${ }^{a}$ \\ ${ }^{a}$ Department of Computer Science, Fault-Tolerant Group at the University Potsdam, P.O. Box 601553, Potsdam 14415, Germany; \\ ${ }^{b}$ Railway-Transportation University of St. Petersburg, pr. Moskovskij 9, Sankt-Petersburg, Russia
}

In this paper we propose a structure dependent method for the systematic design of a selfchecking circuit which is well adapted to the fault model of single gate faults and which can be used in test mode.

According to the fault model considered, maximal groups of independent and unidirectionally independent outputs of an arbitrarily given combinational circuit are determined. A parity bit is added to every group of independent outputs. A few additional outputs are added to every group of unidirectionally independent outputs. In the error free case, these groups of unidirectional independent outputs together with their corresponding additional outputs are elements of a unidirectional error detecting code; for example, a Berger code or an r-out-of-s code.

It is demonstrated how the pairs of (unidirectionally) independent outputs of a given circuit can be determined. A simple heuristic solution for this problem based on a modified circuit graph is also given.

The maximal classes of (unidirectionally) independent outputs can be computed as cliques of a dependency graph where the nodes of the graph are the outputs of the circuit. The applicability of the proposed method is demonstrated for the MCNC benchmarks circuits.

Keywords: Self-Checking circuits, Independent and unidirectionally independent outputs, Parity-code, Berger-code

\section{INTRODUCTION}

The design of self-checking circuits $^{[1-3]}$ and the combination of methods for on-line error detection or concurrent checking and testing is of great interest now (s.e.g. ${ }^{[4]}$ ). It was first proposed in $^{[5]}$ that on-line error detection by duplication and comparison be combined with testing to use the same hardware in different operational modes. These ideas were further developed in ${ }^{[6,7]}$ for an arbitrary prediction function and for independent outputs in ${ }^{[8]}$. Parity prediction for mutually disjoint groups of outputs of combinational circuits is a special case of this approach ${ }^{[9,10]}$. These methods, however, are generally not well-adapted to the specific structure of the monitored circuit and the technical fault model. A method for the design of self-checking and self-testing circuits which is to some extent adapted to the fault model of single gate faults, is given in ${ }^{[8]}$. In that paper, independent

\footnotetext{
* Corresponding author.
} 
outputs of the monitored combinational circuit are the groups of outputs for which the parity is computed, duplicated, inverted, and compared with the parities of the original circuit. In normal operation mode, every single gate fault forcing an output to be erroneous for the first time will be detected. As well, every single gate fault will be detected in test mode. In reality, however, there are only very few cases where all the outputs of a circuit can be combined into groups of independent outputs of a reasonable size which are implemented by completely different gates.

$\mathrm{In}^{[11]}$, weakly independent outputs are used instead of independent outputs for the design of self-testing circuits. Low cost implementations of self-testing circuits are very often possible, but in on-line mode faults may only be detected with some degree of latency and the circuits are not selfchecking.

As a generalization of ${ }^{[8]}$, we propose in this paper a structure dependent method for the systematic design of self-checking combinational circuits which is well-adapted to the fault model of single gate faults and the structure of the considered circuit. Groups of independent outputs as well as groups of unidirectionally independent outputs are determined. Two outputs are unidirectionally independent with respect to a given fault if, in the presence of this fault either both outputs are correct, only one output is erroneous, or both the outputs are unidirectionally erroneous.

In normal operation mode, the proposed circuits are self-checking with respect to all single gate faults. In test mode, these error detection circuits guarantee a $100 \%$ fault coverage for all nonredundant single stuck-at- $0 / 1$ faults.

In this paper, a given combinational circuit is considered. $\mathrm{In}^{[12]}$ a given circuit is modified by use of a special input (output) encoding. The proposed encoding technique guarantees that all internal single stuck-at faults as well as all single stuck-at faults of the input lines will result either in single bit errors or in unidirectional multibit errors of the outputs of the modified circuit. The rest of the paper is organized as follows.
In section 2, the basic notions and notations such as unidirectionally independent outputs, maximal groups of unidirectionally outputs, and generalized circuit graph are given. Basic theorems useful for the determination of independent outputs and unidirectionally independent outputs are also presented. In section 3, it is shown how independent and unidirectionally independent outputs can be used to design self-checking circuits. A few additional outputs are added to every group of unidirectionally independent outputs. In the error free case, these groups of unidirectionally independent outputs are elements of an error detecting code which detects all unidirectional errors. Every group of independent outputs is checked by a parity bit. In section 4, it is explained how pairs of (unidirectionally) independent outputs can actually be determined. The determination of maximal groups of (unidirectionally) independent outputs can be reduced to the standard graph-theoretical problem of the determination of maximal cliques of a dependency graph, where the nodes of the graph are the outputs of the circuit considered.

In section 5 experimental results for benchmark circuits are given.

\section{BASIC NOTIONS AND NOTATIONS}

In this paper we consider a combinational circuit $\mathrm{f}_{c}$ with $n$ binary inputs $x_{1}, \ldots, x_{n}$ and $m$ binary outputs $_{1}=f_{1}\left(x_{1}, \ldots, x_{n}\right), \ldots, y_{m}=f_{m}\left(x_{1}, \ldots, x_{n}\right)$, where $\mathrm{f}_{1}, \ldots, \mathrm{f}_{\mathrm{m}}$ are $\mathrm{n}$-ary Boolean functions. Let $\mathrm{x}=$ $\left(\mathrm{x}_{1}, \ldots, \mathrm{x}_{\mathrm{n}}\right)$, and $\mathrm{y}=\left(\mathrm{y}_{1}, \ldots, \mathrm{y}_{\mathrm{m}}\right)$.

The set of technical faults considered is denoted by $\phi=\left\{\phi_{0}, \phi_{1}, \ldots, \phi_{\mathrm{K}}\right\}$, where $\phi_{0}$ denotes the absence of a fault.

The output $\mathrm{y}_{\mathrm{i}}$ under input $\mathrm{x}$ in the presence of a fault $\phi_{\mathrm{j}} \in \phi$ is described by

$$
\mathrm{y}_{\mathrm{i}, \mathrm{j}}=\mathrm{f}_{\mathrm{i}}\left(\phi_{\mathrm{j}}, \mathrm{x}\right) .
$$

Then, for the correct output in the absence of a fault, we have

$$
\mathrm{y}_{\mathrm{i}, \mathrm{o}}=\mathrm{y}_{\mathrm{i}}=\mathrm{f}_{\mathrm{i}}\left(\phi_{0}, \mathrm{x}\right)=\mathrm{f}_{\mathrm{i}}(\mathrm{x}) .
$$


The notion of unidirectionally independent outputs is introduced in the following definition.

DEFINITION 1 The outputs $y_{i}$ and $y_{k}$ are called unidirectionally independent with respect to the fault $\phi_{\mathrm{j}} \in \phi$ and with respect to a subset $\mathbf{X} \subseteq \mathbf{X}$ of the input set $\mathbf{X}$ if we have, for $\mathrm{x} \in \mathbf{X}$, either

$$
\text { 1. } \mathrm{f}_{\mathrm{i}}(\mathrm{x})=\mathrm{f}_{\mathrm{i}}\left(\phi_{\mathrm{j}}, \mathrm{x}\right) \text { and } \mathrm{f}_{\mathrm{k}}(\mathrm{x})=\mathrm{f}_{\mathrm{k}}\left(\phi_{\mathrm{j}}, \mathrm{x}\right)
$$

or

2. $\left(\mathrm{f}_{\mathrm{i}}(\mathrm{x})=\mathrm{f}_{\mathrm{i}}\left(\phi_{\mathrm{j}}, \mathrm{x}\right)\right.$ and $\left.\mathrm{f}_{\mathrm{k}}(\mathrm{x}) \neq \mathrm{f}_{\mathrm{k}}\left(\phi_{\mathrm{j}}, \mathrm{x}\right)\right)$ or $\left(\mathrm{f}_{\mathrm{i}}(\mathrm{x}) \neq\right.$ $f_{i}\left(\phi_{j}, x\right)$ and $\left.f_{k}(x)=f_{k}\left(\phi_{j}, x\right)\right)$ or

3. $\mathrm{f}_{\mathrm{i}}(\mathrm{x}) \neq \mathrm{f}_{\mathrm{i}}\left(\phi_{\mathrm{j}}, \mathrm{x}\right)$ and $\mathrm{f}_{\mathrm{k}}(\mathrm{x}) \neq \mathrm{f}_{\mathrm{k}}\left(\phi_{\mathrm{j}}, \mathrm{x}\right)$ and $\mathrm{f}_{\mathrm{i}}(\mathrm{x})=$ $f_{k}(x)$.

According to this definition, two outputs are unidirectionally independent if they are both correct, if only one of them is erroneous, or if they are both unidirectionally erroneous. In the last case, both outputs are changing from 0 to 1 or from 1 to 0 .

Independent outputs are a special case of unidirectionally independent outputs. Outputs are independent if only the conditions 1 and 2 of Definition 1 are fulfilled. Thus two outputs are independent if they are either both correct or if only one of them is erroneous at a time.

In the following we sometimes omit the subset $\mathbf{X}$, especially if this subset is not specified. Now we generalize the definition of a pair of unidirectionally independent outputs with respect to a set of faults and a set of outputs.

DEFINITION 2 The outputs $y_{i}$ and $y_{k}$ are called unidirectionally independent with respect to a set $\phi$ of faults if these outputs are unidirectionally independent with respect to every fault $\phi_{\mathrm{j}} \in \phi$.

Definition 3 The outputs $\mathrm{y}_{\mathrm{i}_{1}}, \ldots, \mathrm{y}_{\mathrm{i}_{\mathrm{r}}}$ form a group of unidirectionally independent outputs if every pair of these outputs is unidirectionally independent.

Similar definitions can be given for independent outputs. Independent outputs can be determined by use of the generalized circuit graph introduced in ${ }^{[9]}$. In a similar way, unidirectionally independent outputs can be determined by a modification of this generalized circuit graph. This will be explained now.

A combinational circuit $\mathrm{C}$ can be described by the connections between outputs and inputs of its logical gates. The gates and the direct outputs of the circuit are called "elements" of the circuit. These elements can be combined into maximal classes $C_{i}$ of elements with one output. The maximal classes $C_{i}$ are identified with the nodes $\mathrm{N}_{\mathrm{i}}$ of the generalized circuit graph $\mathrm{G}$. A node $\mathrm{N}_{1}$ of this graph $\mathrm{G}$ is connected with a node $\mathrm{N}_{2}$ by an directed edge directed from $\mathrm{N}_{1}$ to $\mathrm{N}_{2}$ if the output of the class $C_{1}$ is connected to one of the inputs of the gates of the class $\mathrm{C}_{2}$. The maximal classes of elements with one output corresponding to the nodes of the generalized circuit graph can be determined as follows:

1. Create a subset for each non-fanout output of the circuit, and mark each element.

2. If the output of an element is connected only to marked elements of a single, already existing subset, add the element to the subset and mark the element.

3. If the output of an element is connected only to already marked elements not all belonging to the same subset mark this element and create a new subset containing this element. The element is the output of the newly opened subset.

4. Continue until all elements are marked and belong to a subset.

In the next step the circuit graph $\mathrm{G}=(\mathrm{N}, \mathrm{V})$ will be modified into the modified circuit graph $\mathrm{G}^{\prime}=\left(\mathrm{N}^{\prime}, \mathrm{V}^{\prime}\right)$. In addition to the nodes $\mathrm{N}$ of the circuit graph $G$, a node $n_{1}, \ldots, n_{m}$ is assigned to every output $\mathrm{y}_{1}, \ldots, \mathrm{y}_{\mathrm{m}}$ of the circuit $\mathrm{f}_{\mathrm{c}}$. For the set $\mathrm{N}^{\prime}$ of nodes of $\mathrm{G}^{\prime}$, we have $\mathrm{N}^{\prime}=\mathrm{N} \cup\left\{\mathrm{n}_{1}, \ldots, \mathrm{n}_{\mathrm{m}}\right\}$. In the modified circuit graph $G^{\prime}$, the nodes $N_{i}, N_{k} \in$ $\mathrm{N}$ are connected by a non-marked edge if there is a path from the output element of the node $\mathrm{N}_{\mathrm{i}}$ to one of the inputs of the output element of $N_{k}$, with an even number of inversions. The nodes $N_{i}, N_{k} \in N$ are connected by a marked edge if there is a path from the output element of the node $\mathrm{N}_{\mathrm{i}}$ to one of the inputs of the output element of $\mathrm{N}_{\mathrm{k}}$ with an odd 
number of inversions. Inversion of the output of the output element of $\mathrm{N}_{\mathrm{i}}$ as well as inversions at the input of the output element of $\mathrm{N}_{\mathrm{k}}$ are taken into account. Inversions at an input of the output element of $\mathrm{N}_{\mathrm{i}}$ as well as inversions of the output of the output element of $\mathrm{N}_{\mathrm{k}}$ are not considered for the marking of the edge from $\mathrm{N}_{\mathrm{i}}$ to $\mathrm{N}_{\mathrm{k}}$. Thus, two nodes of the modified graph $\mathrm{G}^{\prime}$ are connected by at most two edges, a marked one and an unmarked one.

As an example we consider the combinational circuit of Fig. 1, which implements the following five Boolean functions:

$$
\begin{aligned}
\mathrm{y}_{1}= & \left(\neg \mathrm{x}_{1}\right) \mathrm{x}_{2} \mathrm{x}_{3}\left(\neg \mathrm{x}_{4}\right) \vee\left(\mathrm{x}_{1} \vee \mathrm{x}_{2}\right) \mathrm{x}_{3} \mathrm{x}_{4} \\
& \vee\left(\neg \mathrm{x}_{1}\right)\left(\neg \mathrm{x}_{3}\right) \mathrm{x}_{4} \\
\mathrm{y}_{2}= & \neg\left(\mathrm{x}_{1} \vee \mathrm{x}_{2}\right) \vee \mathrm{x}_{1} \mathrm{x}_{3}\left(\neg \mathrm{x}_{4}\right) \\
& \vee \mathrm{x}_{1}\left(\neg \mathrm{x}_{3}\right) \mathrm{x}_{4} \vee\left(\mathrm{x}_{1} \vee \mathrm{x}_{2}\right)\left(\neg \mathrm{x}_{3}\right)\left(\neg \mathrm{x}_{4}\right) \\
\mathrm{y}_{3}= & \left(\mathrm{x}_{3} \vee \mathrm{x}_{4}\right)\left(\left(\neg \mathrm{x}_{1}\right)\left(\neg \mathrm{x}_{2}\right) \vee\left(\neg \mathrm{x}_{1}\right) \mathrm{x}_{3}\left(\neg \mathrm{x}_{4}\right)\right) \\
\mathrm{y}_{4}= & \left(\neg \mathrm{x}_{1}\right)\left(\neg \mathrm{x}_{2}\right) \vee \mathrm{x}_{1}\left(\neg \mathrm{x}_{3}\right) \mathrm{x}_{4} \vee \mathrm{x}_{1} \mathrm{x}_{3}\left(\neg \mathrm{x}_{4}\right) \\
\mathrm{y}_{5}= & \neg\left(\mathrm{x}_{3} \vee \mathrm{x}_{4}\right) \vee\left(\neg \mathrm{x}_{1}\right) \vee\left(\neg \mathrm{x}_{2}\right) .
\end{aligned}
$$

The modified generalized circuit graph is shown in Fig. 2. According to Fig. 2, two edges are drawn

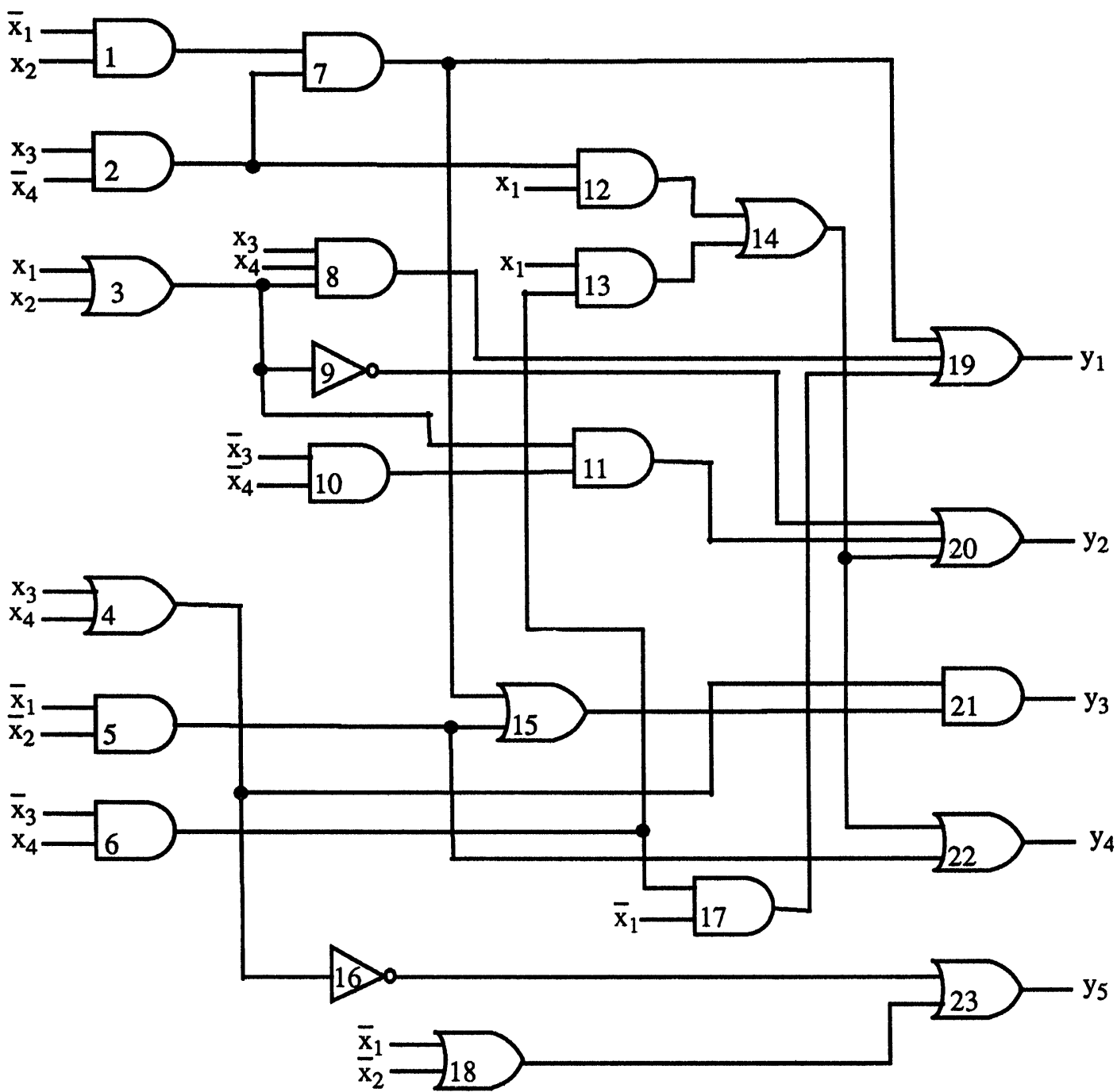

FIGURE 1 Example of a combinational circuit. 


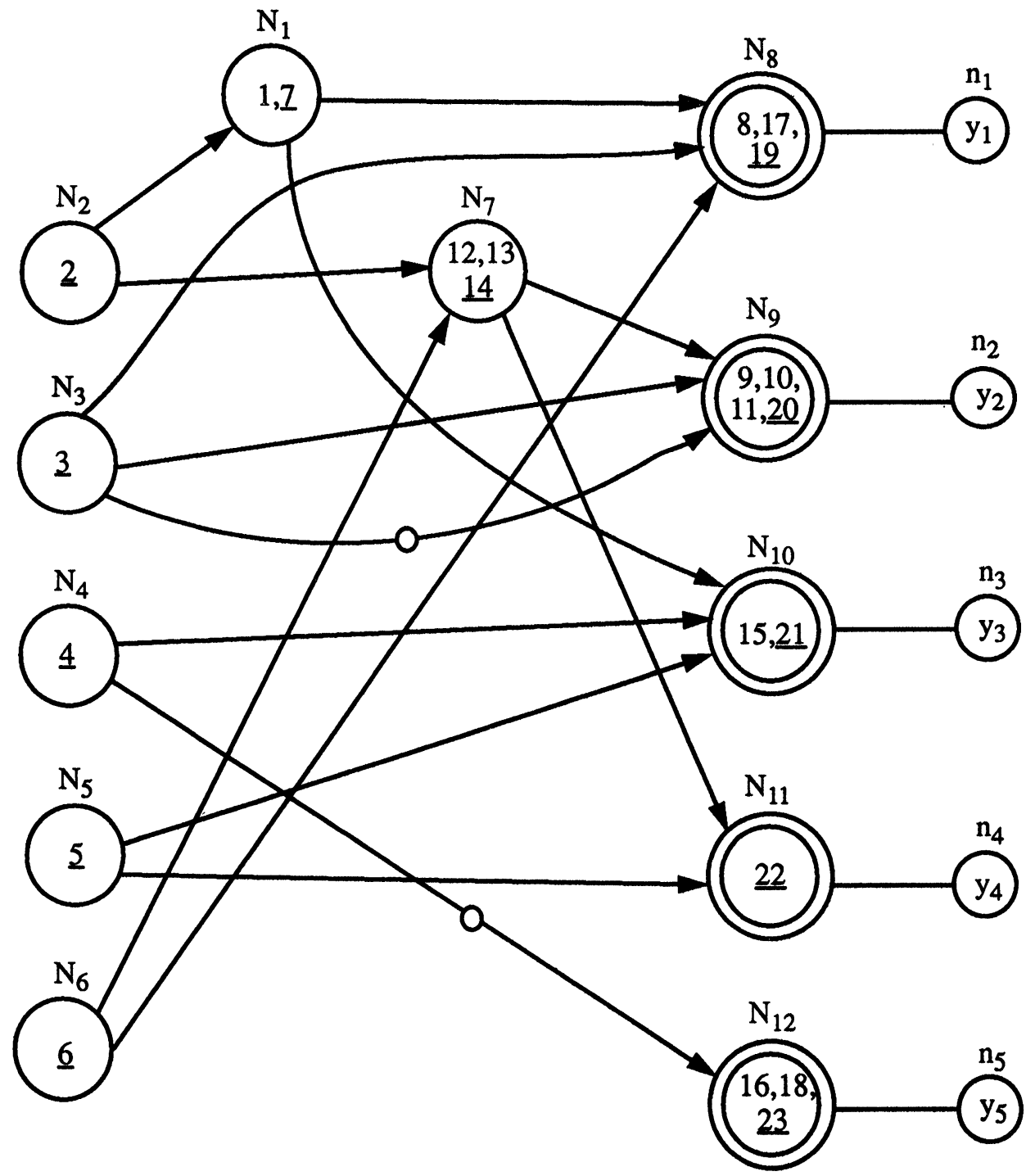

FIGURE 2 Modified generalized circuit graph of the example circuit of Fig. 1.

from the node $\mathrm{N}_{3}$ to the node $\mathrm{N}_{9}$. The first edge corresponds to a path with one inversion (an odd number of inversions) from element 3 to element 20 via element 9 . The second edge via element 11 has no inversion (an even number of inverversions).

Similarly, as in ${ }^{[9]}$, a node $\mathrm{N}_{\mathrm{i}}$ of the modified generalized circuit graph is called structural essential for the node $\mathrm{N}_{\mathrm{k}}$ if there is at least one path from $\mathrm{N}_{\mathrm{i}}$ to $\mathrm{N}_{\mathrm{k}}$. If there is no path from $\mathrm{N}_{\mathrm{i}}$ to $\mathrm{N}_{\mathrm{k}}$ the node $\mathrm{N}_{\mathrm{i}}$ is called structural non-essential for the node $\mathrm{N}_{k}$.
The output of the output element $e_{j}^{r}$ of the maximal class $C_{j}$ corresponding to the node $N_{j}$ will be denoted by $z_{j}$. The upper index $r$ of $e_{j}^{r}$ is the number of the element in the circuit $f_{c}$ if the elements of the circuit are numbered.

We are mainly interested in outputs which are (unidirectionally) independent with respect to functional faults of single gates (single gate faults). The maximal classes of elements with one output are very useful notions to describe these faults. 
DEFINITION 4 The outputs $y_{i}$ and $y_{k}$ are called (unidirectionally) independent with respect to the node $\mathrm{N}_{\mathrm{j}}$ if these outputs are (unidirectionally) independent with respect to all single gate faults within the maximal class $\mathrm{C}_{\mathbf{j}}$.

Proposition 1 The outputs $y_{i}$ and $y_{k}$ are unidirectionally independent with respect to the node $\mathrm{N}_{\mathrm{j}}$ of the generalized circuit graph if and only if we have

$$
F_{i, k ; j}=\frac{d y_{i}}{d z_{j}} \cdot \frac{d y_{k}}{d z_{j}}\left(y_{i} \oplus y_{k}\right)=0
$$

Thereby $z_{j}$ is the output of the output element $e_{j}^{r}$ of the maximal class $C_{j}$ corresponding to the node $N_{j}$.

Proof A fault within the class $\mathrm{C}_{\mathrm{j}}$ which forces an output $y_{i}$ to be erroneous for some $\mathrm{x} \in X$ necessarily changes the output $\mathrm{z}_{\mathrm{j}}(\mathrm{x})$ of $\mathrm{C}_{\mathrm{j}}$ into $\neg \mathrm{z}_{\mathrm{j}}(\mathrm{x})$, and for every input $\mathrm{x} \in X$ there exists a single gate fault within $C_{j}$ which forces $z_{j}(x)$ to be $\neg z_{j}(x)$. (For $z_{j}(x)=1(0)$, a stuck-at- 0 (1) fault of the output gate of $C_{j}$ results in an output of $0(1)$ ).

The inputs of $f_{c}$ for which a change of the output $z_{j}$ of $C_{j}$ results in a change of the output $y_{s}, s=i, k$, are determined by $\mathrm{dy}_{\mathrm{s}} / \mathrm{dz}_{\mathrm{j}}(\mathrm{x})=1$. Thus $\mathrm{dy}_{\mathrm{i}} /$ $d z_{j}(x) \cdot d y_{k} / d z_{j}(x)=1$ determines the inputs for which a change of $z_{j}$ results in a simultaneous change of both the outputs $y_{i}$ and $y_{k}$, respectively. $y_{i}(x) \oplus$ $\mathrm{y}_{\mathrm{k}}(\mathrm{x})=1$ determines the inputs for which we have $\mathrm{y}_{\mathrm{i}}(\mathrm{x})=\neg \mathrm{y}_{\mathrm{k}}(\mathrm{x})$, and $\mathrm{dy}_{\mathrm{i}} / \mathrm{dz}_{\mathrm{j}}(\mathrm{x}) \cdot \mathrm{dy}_{\mathrm{k}} / \mathrm{dz}_{\mathrm{j}}(\mathrm{x})\left(\mathrm{y}_{\mathrm{i}}(\mathrm{x})\right.$ $\left.\oplus \mathbf{y}_{\mathbf{k}}(\mathrm{x})\right)=1$ determines the inputs for which a change of $z_{j}$ simultaneously changes the outputs $\mathrm{y}_{\mathrm{i}}(\mathrm{x})$ and $\mathrm{y}_{\mathrm{k}}(\mathrm{x})$ in different directions. From these equations we conclude (1).

For independent outputs, we have the following proposition.

Proposition 1' The outputs $\mathrm{y}_{\mathrm{i}}$ and $\mathrm{y}_{\mathrm{k}}$ are independent with respect to the node $\mathrm{N}_{\mathrm{j}}$ of the generalized circuit graph if and only if we have

$$
F_{i, k ; j}^{\prime}=\frac{d y_{i}}{d z_{j}} \cdot \frac{d y_{k}}{d z_{j}}=0 .
$$

For practical applications, the following sufficient conditions for the determination of (unidi- rectionally) independent outputs with respect to a node $\mathrm{N}_{\mathrm{j}}$ of the modified generalized circuit graph are of interest.

CONDITION 2 The outputs $\mathrm{y}_{\mathrm{i}}$ and $\mathrm{y}_{\mathrm{k}}$ are unidirectionally independent with respect to the node $\mathrm{N}_{\mathrm{j}}$ if

1. $N_{j}$ is structural non-essential for at least one of the output nodes $n_{i}$ or $n_{k}$,

or

2. all the path's from $N_{j}$ to $n_{i}$ and from $N_{j}$ to $n_{k}$ in the modified generalized Graph $\mathrm{G}^{\prime}$ contain either an even number of marked edges or an odd number of marked edges.

CONDITION $2^{\prime}$ The outputs $y_{i}$ and $y_{k}$ are independent with respect to the node $\mathrm{N}_{\mathrm{j}}$ if $\mathrm{N}_{\mathrm{j}}$ is structural non-essential for at least one of the output nodes $n_{i}$ and $\mathrm{n}_{\mathrm{k}}$.

Example By use of Condition 2, the outputs $\mathrm{y}_{1}$ and $y_{2}$ can be dependent with respect to the node $\mathrm{N}_{3}$, and the outputs $\mathrm{y}_{3}$ and $\mathrm{y}_{5}$ can be dependent with respect to the node $\mathrm{N}_{4}$. To decide whether or not they are unidirectionally independent, we compute

$$
\begin{aligned}
& \frac{\mathrm{dy}_{1}}{\mathrm{dz}_{3}}=\mathrm{x}_{3} \mathrm{x}_{4}, \frac{\mathrm{dy_{2 }}}{\mathrm{dz}}=\left(\neg \mathrm{x}_{1}\right) \mathrm{x}_{3} \vee\left(\neg \mathrm{x}_{1}\right) \mathrm{x}_{4} \vee \mathrm{x}_{3} \mathrm{x}_{4}, \\
& \frac{\mathrm{dy}_{3}}{\mathrm{dz}_{4}}=\left(\neg \mathrm{x}_{1}\right)\left(\neg \mathrm{x}_{2}\right) \vee\left(\neg \mathrm{x}_{1}\right) \mathrm{x}_{3}\left(\neg \mathrm{x}_{4}\right), \frac{\mathrm{dy}_{5}}{\mathrm{dz}}=\mathrm{x}_{1} \mathrm{x}_{2} .
\end{aligned}
$$

Now we check condition 1 :

$$
\begin{gathered}
\frac{d y_{1}}{d z_{3}} \cdot \frac{d y_{2}}{d z_{3}}\left(y_{1} \oplus y_{2}\right)=x_{3} x_{4} \neq 0 \\
\frac{d y_{3}}{d z_{4}} \cdot \frac{d y_{5}}{d z_{4}}\left(y_{3} \oplus y_{5}\right)=0 .
\end{gathered}
$$

Thus the outputs $\mathrm{y}_{3}$ and $\mathrm{y}_{5}$ are unidirectionally independent and the outputs $y_{1}$ and $y_{2}$ are not.

The following theorem is of great practical interest for the determination of unidirectionally independent outputs.

Proposition 3 Two outputs $y_{i}$ and $y_{k}$ are (unidirectionally) independent with respect to the set of all single gate faults $\phi$ if they are (unidirectionally) independent with respect to all nodes $\mathrm{N}_{\mathrm{j}}$ of the generalized circuit graph. 


\section{DESIGN OF SELF-CHECKING CIRCUITS WITH UNIDIRECTIONALLY INDEPENDENT OUTPUTS}

Figure 3 shows the general structure of the proposed self-checking circuit.

The outputs $y_{1}, \ldots, y_{m}$ of the circuit $f_{c}$ are arranged in $h$ different groups $Y_{1}, \ldots, Y_{h}$ with $Y_{i}=\left\{y_{i_{1}}, \ldots, y_{i_{n i}}\right\}, i=1, \ldots, h$, of independent outputs and unidirectionally independent outputs. Every group $\mathrm{Y}_{\mathrm{i}}=\left\{\mathrm{y}_{\mathrm{i}_{1}}, \ldots, \mathrm{y}_{\mathrm{i}_{\mathrm{n}}}\right\}, \mathrm{i}=1, \ldots, \mathrm{h}$, will be supplemented with some additional outputs $\mathrm{v}_{\mathrm{i}_{1}}, \ldots, \mathrm{v}_{\mathrm{i}_{\mathrm{i}}}$ forming an extended group $\mathrm{Y}_{\mathrm{i}}=$ $\left\{\mathrm{y}_{\mathrm{i}_{1}}, \ldots, \mathrm{y}_{\mathrm{i}_{\mathrm{in}}}, \mathrm{v}_{\mathrm{h}_{1}}, \ldots, \mathrm{v}_{\mathrm{h}_{\mathrm{ti}}}\right\}$.

If the outputs of a group $Y_{j}$ are independent only a single parity bit $v_{j 1}$ is added. In Fig. $3 Y_{1}$ is assumed to be a group of independent outputs. Therefore only a single parity bit is added to this group.

If the outputs of a group $Y_{i}$ are unidirectionally independent the $t_{i}$ additional outputs are determined in such a way that the outputs $\mathrm{y}_{\mathrm{i}_{1}}, \ldots, \mathrm{y}_{\mathrm{i}_{\mathrm{n}}}$, $\mathrm{v}_{\mathrm{h}_{1}}, \ldots, \mathrm{v}_{\mathrm{h}_{\mathrm{i}}}$ are elements of an error detecting code for all unidirectional errors.

In Fig. 3 the group $Y_{h}$ is supposed to be a group of unidirectionally independent outputs. Thus the outputs $\mathrm{v}_{\mathrm{h}_{1}}, \ldots, \mathrm{v}_{\mathrm{h}_{\mathrm{ti}}}$ are added.

A possible error detecting code is a Berger$\operatorname{code}^{[13]}$ or an s-out-of-r code $e^{[14]}$ where $s$ is the number of 1's and $r$ is the number of bits, $r=n_{i}+t_{i}$.

To determine the elements of an s-out-of-r code we consider all the possible binary $\mathrm{n}_{\mathrm{i}}$-tupels of the

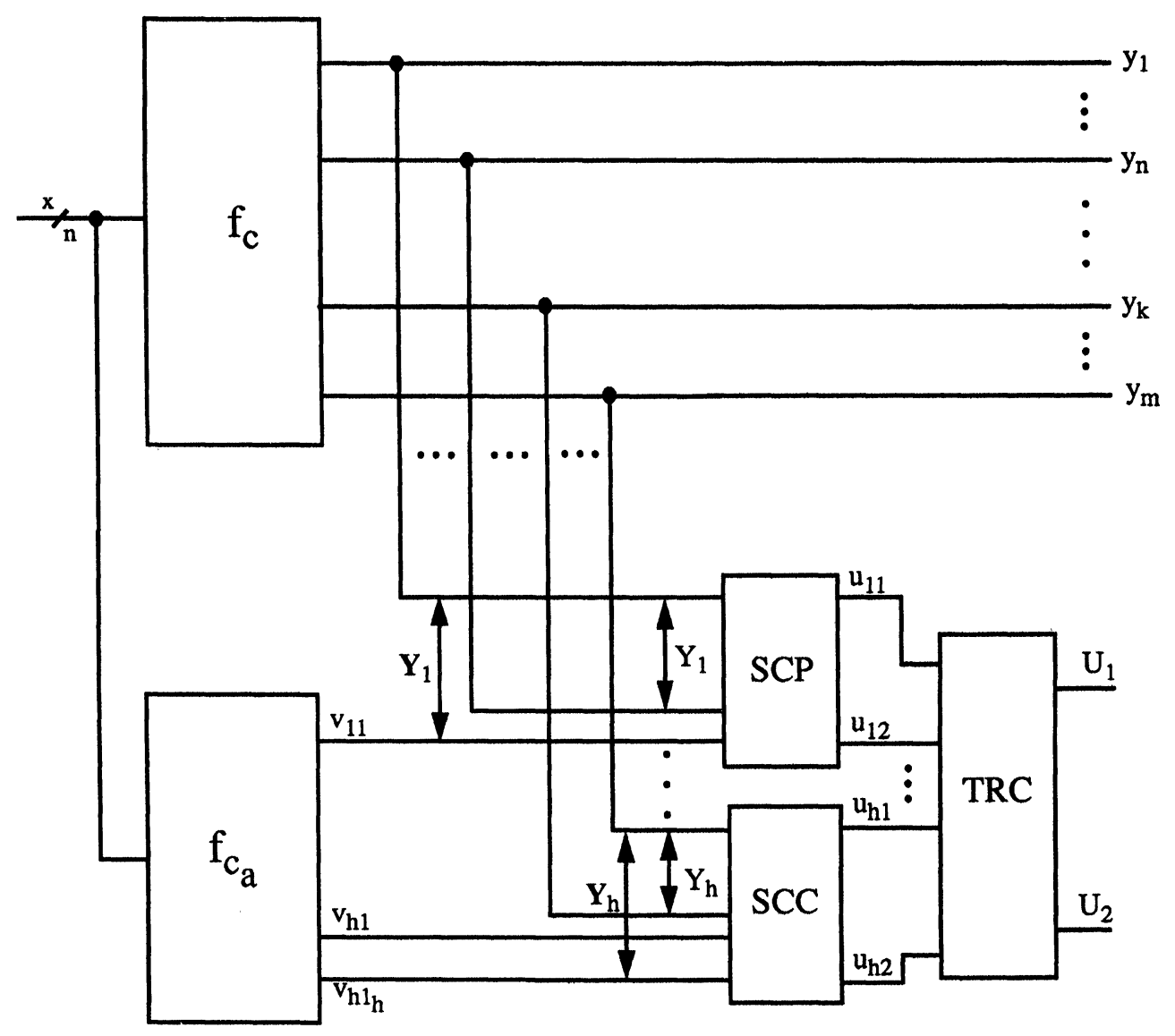

FIGURE 3 General structure of the proposed self-checking circuit. 
outputs $y_{i_{1}}, \ldots, y_{i_{n i}}$ of the group $Y_{i}$ for $x \in X$. Let $M_{i}\left(m_{i}\right)$ be the maximal (minimal) number of 1 's of this n-tupels. Then $t_{i}$ is determined by $t_{i}=M_{i}-m_{i}$. It is easy to determine the outputs $v_{h_{1}} \ldots v_{h_{t i}}$ such that $\mathrm{y}_{\mathrm{i}_{1}} \ldots \mathrm{y}_{\mathrm{i}_{\mathrm{n}}}, \mathrm{v}_{\mathrm{h}_{1}} \ldots \mathrm{v}_{\mathrm{h}_{\mathrm{ti}}}$ are elements of an $\mathrm{M}_{\mathrm{i}}$-outof- $\left(n_{i}+t_{i}\right)$ code.

If the number of 1 's of the considered $n_{i}$-tuple $\mathrm{y}_{\mathrm{i} 1}, \ldots, \mathrm{y}_{\mathrm{ini}}$ is $\mathrm{K}_{\mathrm{i}}, \mathrm{m}_{\mathrm{i}} \leq \mathrm{K}_{\mathrm{i}} \leq \mathrm{M}_{\mathrm{i}}$, then exactly $\mathrm{M}_{\mathrm{i}}$ $\mathrm{K}_{\mathrm{i}}$ additional outputs $\mathrm{v}_{\mathrm{h} 1}, \ldots, \mathrm{v}_{\mathrm{hti}}$ have to be 1 . These $\mathbf{M}_{\mathbf{i}}-\mathbf{K}_{\mathbf{i}}$ ones can be arbitrarily distributed over the additional outputs $\mathrm{v}_{\mathrm{h} 1}, \ldots, \mathrm{v}_{\mathrm{hti}}$.

These additional outputs will be jointly implemented by use of an additional combinational circuit $f_{c a}$ as shown in Fig. 3. Every fault in the additional circuit $\mathrm{f}_{\mathrm{ca}}$ will be detected.

Every extended group $\mathbf{Y}_{\mathrm{i}}$ is checked by use of a self-checking code-checker ${ }^{[2,3,15,16]}$ with two outputs $u_{i_{1}}, u_{i_{2}}$ with $u_{i_{1}}=\neg u_{i_{2}}$ in the fault free case. These outputs are compacted by a two-rail checker TRC with $2 \mathrm{~h}$ inputs and 2 outputs $\mathrm{U}_{1}$ and $\mathrm{U}_{2}$, with $\mathrm{U}_{1}=\neg \mathrm{U}_{2}$ in the fault free case. Every single gate fault of the circuit $f_{c}$ and every fault of the circuit $f_{c a}$ will be detected by an output $u_{i_{1}}=u_{i_{2}}$ for some $i$ if it forces at least one of the outputs of $f_{c}$ or $f_{c a}$ to be erroneous for the first time. Since $u_{i_{1}}=u_{i_{2}}$ implies $\mathrm{U}_{1}=\mathrm{U}_{2}$ at the output of the two-rail checker, every single gate fault of $f_{c}$ and $f_{c a}$ will be immediately detected. In Fig. 3 the group $Y_{1}$ is checked by a selfchecking parity checker SCP, and the group $\mathbf{Y}_{\mathrm{h}}$ is checked by a self-checking code checker SCC for the corresponding code.

\section{DETERMINATION OF INDEPENDENT AND UNIDIRECTIONALLY INDEPENDENT OUTPUTS}

Pairs of independent outputs can be determined in the following steps:

1. Consider the nodes $\mathrm{n}_{1}, \ldots, \mathrm{n}_{\mathrm{m}}$ of $\mathrm{f}_{\mathrm{c}}$. To every node $n_{j}, j=1, \ldots, m$, we assign a set $S_{j}$. Thereby a node $N_{k}, N_{k} \in N$ belongs to $S_{j}$ if there exists a path from $N_{k}$ to $n_{j}$.

2. For every pair of nodes $n_{i}, n_{j}$ with $i \neq j$, $i, j=1, \ldots, n$, we determine the set $R_{i, j}=S_{i} \cap S_{j}$.
If $R_{i, j}=\emptyset$ the outputs $y_{i}$ and $y_{j}$ are obviously independent. If $R_{i, j} \neq \emptyset$ we have to compute the expression $F_{i, j ; k}^{\prime}$ according to $\left(1^{\prime}\right)$. If for all $k$ with $N_{k} \in R_{i, j}, R_{i, j} \neq \emptyset$ we have $F_{i^{\prime}, j ; k}=0$, the outputs $y_{i}$ and $y_{j}$ are independent.

For the determination of pairs of unidirectionally independent outputs this procedure has to be modified.

$1^{\prime}$. To every node $n_{j}, j=1, \ldots, m$, we assign two sets $T_{j}$ and $\left.T_{j}\right\urcorner$. A node $N_{k}, N_{k} \in N$, belongs to $T_{j}\left(T_{j}^{\urcorner}\right)$if there exist a path from $N_{k}$ to $n_{j}$ with an even (odd) number of inversions.

$2^{\prime}$. For every pair of nodes $n_{i}, n_{j}$ with $i \neq j$, $i, j=1, \ldots, n$, we determine the set $M_{i, j}=T_{i} \cap$ $\left.\left.\left(T_{j}\right\urcorner\right) \cup T_{j} \cap\left(T_{i}\right\urcorner\right)$. If $M_{i, j}=\emptyset$, the outputs $y_{i}$ and $y_{j}$ are obviously unidirectionally independent and the outputs $y_{i}$ and $y_{j}$ are called graphically unidirectionally independent. If $\mathbf{M}_{\mathrm{i}, \mathrm{j}} \neq \emptyset$ we have to compute the expression $F_{i, j ; k}$ according to (1).

If for all $k$ with $N_{k} \in M_{i, j}, M_{i, j} \neq \emptyset$ we have $F_{i, j ; k}=0$, the outputs $y_{i}$ and $y_{j}$ are unidirectionally independent.

For the circuit $f_{c}$ of Fig. 1 with the generalized circuit graph of Fig. 2, we have

$$
\begin{aligned}
& S_{1}=\left\{N_{1}, N_{2}, N_{3}, N_{6}, N_{8}\right\}, \\
& S_{2}=\left\{N_{2}, N_{3}, N_{6}, N_{7}, N_{9}\right\}, \\
& S_{3}=\left\{N_{1}, N_{2}, N_{4}, N_{5}, N_{10}\right\}, \\
& S_{4}=\left\{N_{2}, N_{5}, N_{6}, N_{7}, N_{11}\right\}, \\
& S_{5}=\left\{N_{4}, N_{12}\right\}
\end{aligned}
$$

from which we conclude that

$$
\begin{aligned}
& \mathbf{R}_{1,5}=S_{1} \cap S_{5}=\emptyset, R_{2,5}=S_{2} \cap S_{5}=\emptyset, \\
& R_{4,5}=S_{4} \cap S_{5}=\emptyset,
\end{aligned}
$$

and the pairs of outputs $\mathrm{y}_{1}$ and $\mathrm{y}_{5}, \mathrm{y}_{2}$ and $\mathrm{y}_{5}$ and $\mathrm{y}_{4}$ and $\mathrm{y}_{5}$ are graphically independent.

For $\mathrm{j}=1, \ldots, 5$ the sets

$$
T_{j}\left(T_{j}^{\neg}\right)
$$


are

$$
\begin{aligned}
& \mathrm{T}_{1}=\left\{\mathrm{N}_{1}, \mathrm{~N}_{2}, \mathrm{~N}_{3}, \mathrm{~N}_{6}, \mathrm{~N}_{8}\right\}, \mathrm{T}_{1}^{\urcorner}=\emptyset, \\
& \mathrm{T}_{2}=\left\{\mathrm{N}_{2}, \mathrm{~N}_{3}, \mathrm{~N}_{6}, \mathrm{~N}_{7}, \mathrm{~N}_{9}\right\}, \mathrm{T}_{2}^{\urcorner}=\left\{\mathrm{N}_{3}\right\} \\
& \mathrm{T}_{3}=\left\{\mathrm{N}_{1}, \mathrm{~N}_{2}, \mathrm{~N}_{4}, \mathrm{~N}_{5}, \mathrm{~N}_{10}\right\}, \mathrm{T}_{3}^{\urcorner}=\emptyset \\
& \mathrm{T}_{4}=\left\{\mathrm{N}_{2}, \mathrm{~N}_{5}, \mathrm{~N}_{6}, \mathrm{~N}_{7}, \mathrm{~N}_{11}\right\}, \mathrm{T}_{4}^{\urcorner}=\emptyset \\
& \mathrm{T}_{5}=\left\{\mathrm{N}_{12}\right\}, \mathrm{T}_{5}=\left\{\mathrm{N}_{4}\right\} .
\end{aligned}
$$

For

$$
M_{i, j}, i \neq j, i, j=1, \ldots, 5
$$

we have

$\mathbf{M}_{12}=\left\{\mathbf{N}_{3}\right\}, \mathbf{M}_{13}=\emptyset, \mathbf{M}_{14}=\emptyset, \mathbf{M}_{15}=\emptyset, \mathbf{M}_{23}=\emptyset$, $\mathbf{M}_{24}=\emptyset, \mathbf{M}_{25}=\emptyset, \mathbf{M}_{34}=\emptyset, \mathbf{M}_{35}=\left\{\mathbf{N}_{4}\right\}, \mathbf{M}_{45}=\emptyset$

and the pairs of output nodes

$$
\begin{aligned}
& \left(\mathrm{n}_{1}, \mathrm{n}_{3}\right),\left(\mathrm{n}_{1}, \mathrm{n}_{4}\right),\left(\mathrm{n}_{1}, \mathrm{n}_{5}\right),\left(\mathrm{n}_{2}, \mathrm{n}_{3}\right),\left(\mathrm{n}_{2}, \mathrm{n}_{4}\right),\left(\mathrm{n}_{2}, \mathrm{n}_{5}\right), \\
& \left(\mathrm{n}_{3}, \mathrm{n}_{4}\right) \text { and }\left(\mathrm{n}_{4}, \mathrm{n}_{5}\right)
\end{aligned}
$$

are graphically unidirectionally independent.

If we would like to determine not only the graphically unidirectionally independent pairs of outputs, the cases $\mathbf{M}_{i, j} \neq \emptyset$, i.e. $\mathbf{M}_{1,2}=\left\{\mathbf{N}_{3}\right\}$ and $\mathbf{M}_{3,5}=\left\{\mathrm{N}_{4}\right\}$ have to be considered in more detail.

Since we have

$$
F_{1,2 ; 3}=\frac{d_{1}}{d z_{3}}(x) \cdot \frac{d_{2}}{d z_{3}}(x)\left(y_{1}(x) \oplus y_{2}(x)\right)=x_{3} x_{4} \neq 0
$$

and

$$
\mathrm{F}_{3,5 ; 4}=\frac{\mathrm{dy}_{3}}{\mathrm{dz}}(\mathrm{x}) \cdot \frac{\mathrm{dy}_{5}}{\mathrm{dz}}(\mathrm{x})\left(\mathrm{y}_{3}(\mathrm{x}) \oplus \mathrm{y}_{5}(\mathrm{x})\right)=0,
$$

the outputs $y_{3}$ and $y_{5}$ are unidirectionally independent and the outputs $\mathrm{y}_{1}$ and $\mathrm{y}_{2}$ are not unidirectionally independent.

Now we describe how (maximal) sets of independent outputs can be determined. To this end we draw a first dependency graph $\underline{G}^{1}=\left(\underline{N}^{1}, \underline{V}^{1}\right)$. The nodes $\underline{N}^{1}$ of $\underline{G}^{1}$ are the outputs $y_{1}, \ldots, y_{n}$ of the considered circuit $f_{c}$. Two nodes $y_{i}, y_{j} \in \underline{N}^{1}$ are connected by an edge if the pair of nodes is (graphically) independent. Every group of independent outputs corresponds to a complete subgraph of $\underline{G}^{1}$, i.e. a clique of $\underline{G}^{1}$. The determination of a complete subgraph is a standard problem of graph theory.
In a similar way, we determine sets of unidirectionally independent outputs. We draw a second dependency graph $\underline{G}^{2}=\left(\underline{N}^{2}, \underline{V}^{2}\right)$. The nodes $\underline{N}^{2}$ of $\mathrm{G}^{2}$ are again the outputs $\mathrm{y}_{1}, \ldots, \mathrm{y}_{\mathrm{n}}$ of the considered circuit $\mathrm{f}_{\mathrm{c}}$. Two nodes $\mathrm{y}_{\mathrm{i}}, \mathrm{y}_{\mathrm{j}} \in \mathrm{N}^{2}$ are connected by an edge if these nodes are (graphically) unidirectionally independent. Every group of unidirectionally independent outputs corresponds to a complete subgraph of $\underline{G}^{2}$, i.e. a clique of $\underline{G}^{2}$. In the following example we restrict ourself to the graphically independent and graphically unidirectionally independent outputs.

The graph $\underline{G}^{1}$ of graphically independent pairs of outputs of $f_{c}$ is given in Fig. 4. The three possible maximal complete subgraphs consist of the nodes $\mathrm{n}_{1}$ and $\mathrm{n}_{5}, \mathrm{n}_{2}$ and $\mathrm{n}_{5}$ and $\mathrm{n}_{4}$ and $\mathrm{n}_{5}$. Fig. 5 shows the graph $\underline{G}^{2}$ of the graphically unidirectionally

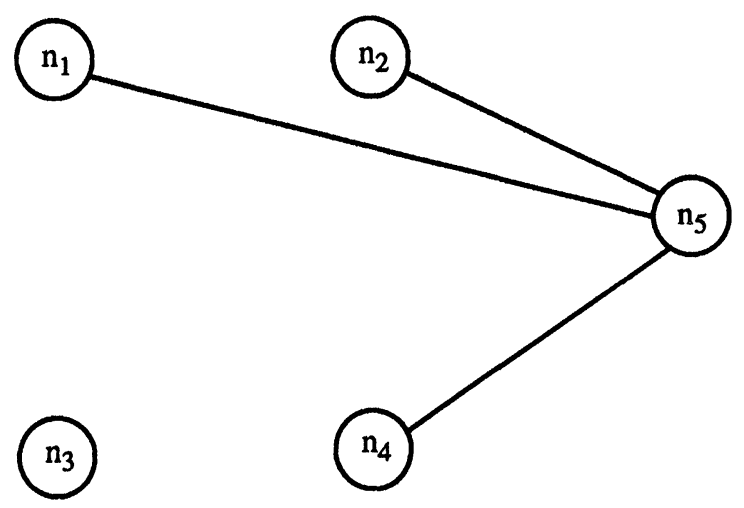

FIGURE 4 Graph of independent outputs.

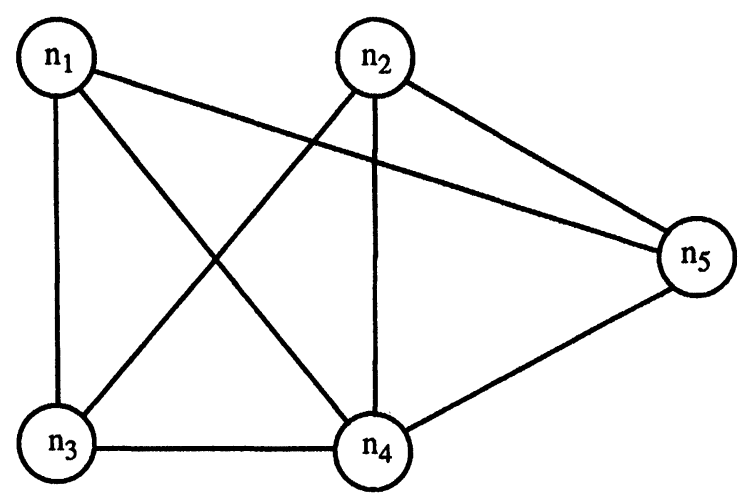

FIGURE 5 Graph of unidirectionally independent outputs. 


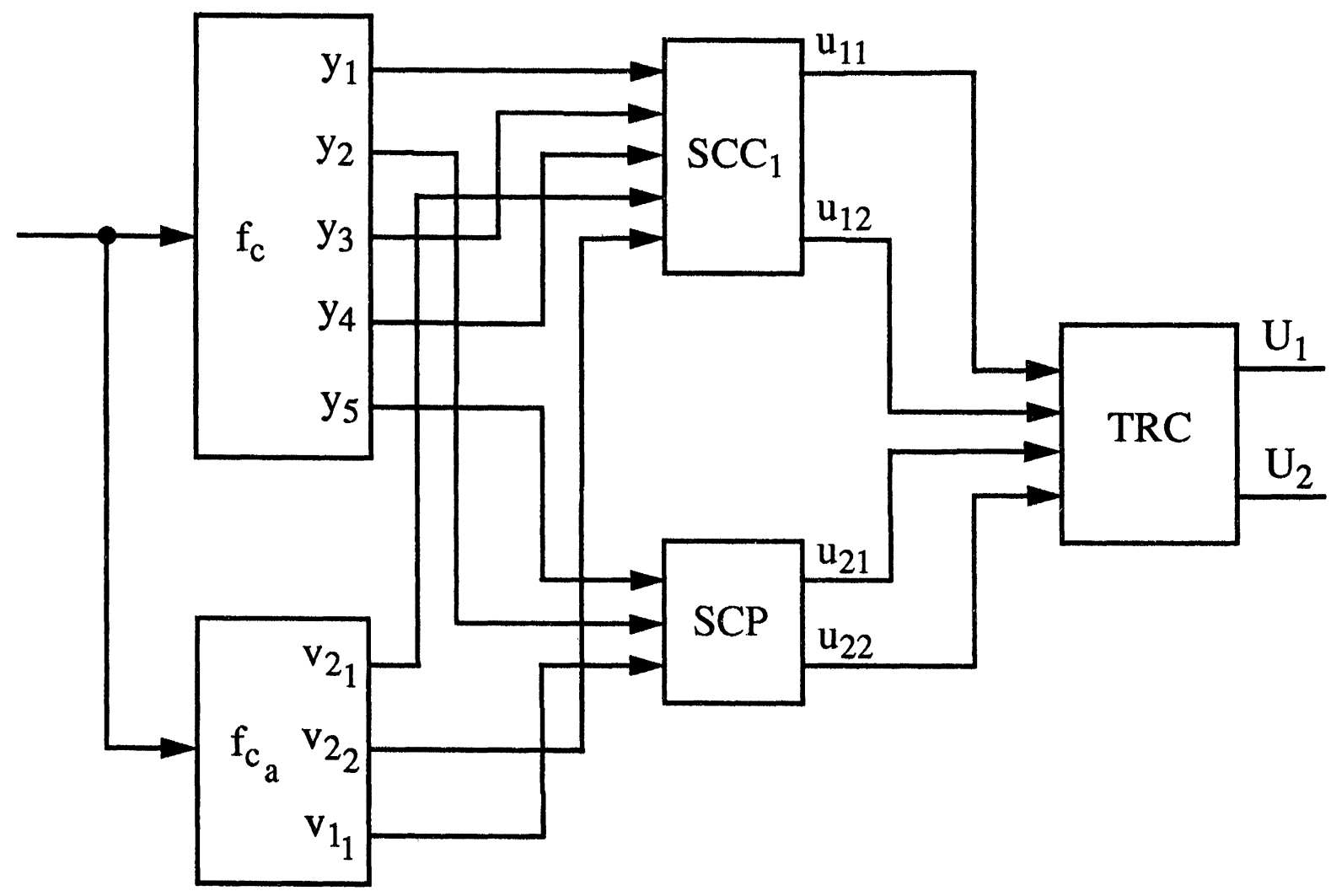

FIGURE 6 Self-checking circuit of the example of Fig. 1.

independent outputs of $f_{c}$. The maximal complete subgraphs of $\underline{G}^{2}$ consist of the sets of nodes $\left\{\mathrm{n}_{1}, \mathrm{n}_{4}, \mathrm{n}_{5}\right\},\left\{\mathrm{n}_{1}, \mathrm{n}_{3}, \mathrm{n}_{4}\right\},\left\{\mathrm{n}_{2}, \mathrm{n}_{3}, \mathrm{n}_{4}\right\},\left\{\mathrm{n}_{3}, \mathrm{n}_{4}, \mathrm{n}_{5}\right\}$ and $\left\{\mathrm{n}_{2}, \mathrm{n}_{4}, \mathrm{n}_{5}\right\}$.

We choose the set $Y_{1}=\left\{n_{2}, n_{5}\right\}$ of graphically independent outputs and the set $Y_{2}=\left\{n_{1}, n_{3}, n_{4}\right\}$ of graphically unidirectionally independent outputs. To the set $\mathrm{Y}_{1}=\left\{\mathrm{y}_{2}, \mathrm{y}_{5}\right\}$ of graphically independent outputs a single parity bit $\mathrm{v}_{1_{1}}, \mathrm{v}_{1_{1}}=\neg\left(\mathrm{y}_{2} \oplus \mathrm{y}_{5}\right)$ is added. Thus we have $Y_{1}=\left\{y_{2}, y_{5}, v_{1_{1}}\right\}$.

To the outputs $\mathrm{y}_{1}, \mathrm{y}_{3}, \mathrm{y}_{4}$ of the group $\mathbf{Y}_{2}$ two additional outputs $\mathrm{v}_{2_{1}}, \mathrm{v}_{2_{2}}$ are added to form a Beger code with three information bits and two control bits. All the additional outputs $\mathrm{v}_{1_{1}}, \mathrm{v}_{2_{1}}$ and $\mathrm{v}_{2_{2}}$ can be jointly implemented. The outputs $\mathrm{y}_{2}, \mathrm{y}_{5}$, and $\mathbf{v}_{1}$ of the group $\mathbf{Y}_{1}$ are checked by a selfchecking parity checker SCP. The group $Y_{2}$ is checked by a self-checking Berger code checker
$\mathrm{SCC}_{1}$. The outputs of the $\mathrm{SCC}_{1}$ and the SCP are compacted by a self-checking two-rail-checker TRC as shown in Fig. 6.

The resulting circuit is self-checking.

\section{EXPERIMENTAL RESULTS}

Table 1 showes the experimental results for the MCNC benchmarks.

The following abbreviations are used:

inp: number of inputs

out: number of outputs

pgio: number of pairs of graphically independent outputs

pguo: number of pairs of graphically unidirectionally independent outputs

pio: number of pairs of independent outputs 
TABLE I Experimental results

\begin{tabular}{lrrrrrrrrrrr}
\hline circuit & inp & out & pgio & pguo & pio & puo & go & bB & bP & bT & ao \\
\hline 1 & 2 & 3 & 4 & 5 & 6 & 7 & 8 & 9 & 10 & 11 & 12 \\
cm138a & 6 & 8 & - & 28 & - & 28 & 1 & 4 & - & 4 & 15 \\
cu & 14 & 11 & 33 & 54 & 33 & 54 & 2 & 5 & - & 5 & 75 \\
cm162a & 14 & 5 & - & 3 & - & 4 & 4 & 5 & - & 5 & 107 \\
decod & 5 & 16 & 27 & 120 & 27 & 120 & 1 & 5 & - & 5 & $<1$ \\
ldd & 9 & 19 & 29 & 79 & 61 & 171 & 1 & 5 & - & 5 & 31 \\
apex7 & 49 & 37 & 361 & 522 & 379 & 555 & 12 & 3 & 10 & 13 & 119 \\
z4m1 & 7 & 4 & - & - & 3 & 6 & 3 & - & 3 & 3 & 62 \\
x2 & 10 & 7 & 3 & 14 & 4 & 15 & 4 & 2 & 3 & 5 & 85 \\
c8 & 28 & 18 & 96 & 152 & 96 & 152 & 2 & 6 & - & 6 & 156 \\
f51m & 8 & 8 & 1 & 5 & 1 & 7 & 4 & 6 & 1 & 7 & 55 \\
ttt2 & 24 & 21 & 105 & 116 & 128 & 148 & 7 & 4 & 5 & 9 & 79 \\
vda & 17 & 39 & 11 & 17 & 169 & 343 & 14 & 26 & - & 26 & 60 \\
pm1 & 16 & 13 & 63 & 68 & 69 & 74 & 2 & 3 & 1 & 4 & 88 \\
\hline
\end{tabular}

puo: number of pairs of unidirectionally independent outputs

go: number of groups of independent and unidirectionally independent outputs

bB: number of additional bits for Berger Codes

bP: number of additional parity bits

bT: total number of additional bits $(\mathrm{bT}=$ $\mathrm{bB}+\mathrm{bP})$

ao: additional area overhead in percent of the area of the original circuit

The pairs of graphically independent outputs pgio (column 4) and the pairs of graphically unidirectionally independent outputs pguo (column 5) are determined by use of the modified generalized circuit graph. The pairs of independent outputs pio (column 6) and the pairs of unidirectionally independent outputs puo (column 7) are determined according to (1) and $\left(1^{\prime}\right)$ respectively where the corresponding Boolean derivations have to be taken into account.

The Boolean derivations in (1) and $\left(1^{\prime}\right)$ are computed by use of the program system MIS ${ }^{[17]}$. The corresponding Boolean functions are represented as BDD's. Graphically (unidirectionally) independent outputs are always (unidirectionally) independent. Therefore, the number of pairs of (unidirectionally) independent outputs is always greater than or equal to the number of pairs of graphically (unidirectionally) independent outputs.
The minimal number of mutually disjoint groups of independent and unidirectionally independent outputs go are given in column 8 .

Groups of unidirectionally independent outputs are monitored by Berger Codes. The number of necessary additional bits $b B$ is represented in column 9. Groups of independent outputs are checked by additional parity bits. The number of additional parity bits bP is given in column 10 . The total number of check bits bT (Berger code bits and parity bits) is given in column 11 of Table I. The area overhead of the additional circuit $\mathrm{f}_{\mathrm{c}_{\mathrm{a}}}$ is shown in the row 12 of Table. The additional circuit $f_{c_{a}}$ was sythesized by use of the structurally given circuit $f_{c}$ in the following way: If the outputs of a group $Y_{i}=\left\{y_{i_{1}}, \ldots, y_{i_{h i}}\right\}$ are independent a single additional output $v_{i_{1}}$ of the parity bit is determined by connecting the outputs $\mathrm{y}_{\mathrm{i}_{1}}, \ldots, \mathrm{y}_{\mathrm{i}_{\mathrm{hi}}}$ by XOR-gates. If the outputs of a group $\mathrm{Y}_{\mathrm{j}}=\left\{\mathrm{y}_{\mathrm{j}_{1}}, \ldots, \mathrm{y}_{\mathrm{j}_{\mathrm{hj}}}\right\}$ are unidirectionally independent the outputs $\left\{\mathrm{y}_{\mathrm{j}_{1}}, \ldots, \mathrm{y}_{\mathrm{j}_{\mathrm{hj}}}\right\}$ are connected to a counter of zeros to determine the additional bits of a Berger code. Then all the outputs of the circuit $f_{c_{a}}$ are jointly optimized by use of the standard MIS-algorithm script.rugget.

The necessary computing time on a SUN Workstation Spark 10 including the determination of pairs of independent and unidirectionally independent outputs, the determination of maximal complete subgraphs and the synthesis of the additional 
circuit was in the range of 0.33 to 3376.46 seconds. The average additional area overhead is $71 \%$.

\section{CONCLUSIONS}

In this paper a new structure dependent method for the design of self-checking and self-testing combinational circuits was proposed. Maximal groups of independent outputs and unidirectionally independent outputs are determined. It is shown how pairs of (unidirectionally) independent outputs can heuristically be determined by a simple graphtheoretical algorithm. Maximal groups of (unidirectionally) independent outputs can be computed as cliques of a dependency graph, which is a standard graph-theoretical problem. A single parity bit is added to every group of independent outputs. A few additional outputs are added to every group of unidirectionally independent outputs in such a way that these groups of unidirectionally independent outputs and the corresponding additional outputs are elements of a unidirectional error detecting code. This method is explained in detail for a simple example. The applicability of the proposed method is demonstrated for the MCNC benchmarks.

\section{Acknowledgment}

The authors are very grateful to the reviewers of this paper for their helpful comments.

\section{References}

[1] P. K. Lala, Fault Tolerant and Fault Testable Hardware Design. Prentice Hall, Englewood-Cliffs, N.J.

[2] V. V. Sapozhnikov and Vl. V. Sapozhnikov, Self-Checking Checkers for Balanced Codes. Automation and Remote Control, vol. 53, No 3, part 1, 321-348 (1992).

[3] V. V. Saposhnikov and V1. V. Saposhnikov, Self-Checking Discrete Circuits (in russ.) Energoatomisdat, St. Petersburg (1992).

[4] S. K. Gupta and D. K. Pradhan, Can Concuent Checkers Help BIST?, Proc. 1992 Intern. Test Conference, pp. 140-150 (1992).

[5] R. M. Sedmak, Design for Self-Verification. An Approach for Dealing with Testability Problems in VLSI-based Design. Proc. 1979 IEEE Test Conference, pp. 112-120 (1979).
[6] E. Fujiwara, N. Muto and K. Matsuoka, A Self-Testing Group Parity Prediction Checker and its Use for Built-inTesting. IEEE Trans. Comp., vol. C-33, No 6, 578-583 (1984).

[7] T. R. N. Rao and E. Fujiwara, Error Control Coding for Computer Systems, Prentice Hall (1989).

[8] E. S. Sogomonyan, Reliability of Self-Testing using Functional Diagnosic Tools. Automation and Remote Control, vol. 49, No 10, Part 2, October 1988, pp. 13761380.

[9] E. S. Sogomonyan, Design of Built-In Self-Checking Monitoring Circuits for Combinational Devices. Automation and Remote Control, vol. 35, No 2, part 2, 280-289 (1974).

[10] E. Fujiwara, A Self-Testing Group Parity Prediction Checker and its Use for Built-in Testing. Proc. 13th Symposium Fault-Tolerant Computing, Milano, pp. 146153 (1983).

[11] E. S. Sogomonyan and M. Goessel, Design of Self-Testing and On-line Fault Detection Combinational Circuits with Weakly Independent Outputs. JETTA, 4, 267-281 (1993).

[12] F. Y. Busaba and P. K. Lala, Self-Checking Combinational Circuit Design for Single and Unidirectional Multibit Errors, JETTA, 5, 19-28 (1994).

[13] J. M. Berger, A Note on Error Detecting Codes for Asymmetric Channels. Information and Control, 4, 68-73 (1991).

[14] D. A. Anderson and G. Metze, Design of Totally SelfChecking Check Circuits for m-out-of-n Codes. IEEE Trans. Comp., vol. C-22, 263-269 (1973).

[15] M. A. Marouf and A. D. Friedman, Design of SelfChecking Checkers for Berger-Codes. Proc. Int. Symp. Fault-Tolerant Computing, 179-184 (1978).

[16] S. Kundu and S. M. Reddy, Embedded Totally SelfChecking Checkers. A Practical Design. IEEE Design and Test of Computers, August 1990, 5-16.

[17] R. K. Brayton, R. Rudell, A. Sangiovanni-Vincentelli and A. R. Wang, "MIS: A multi-level logic optimization program," IEEE Trans. Computer-Aided Design, 7, 1062-1081.

\section{Authors' Biographies}

Andrej Morosow received the M.S. degree in electrical enigneering from the University of Railway Engineering, St. Petersburg, Russia, in 1992. From 1992 to 1996 he was with Max-PlanckSociety Fault Tolerant Computing Group at the University Potsdam, Germany. Now he is with the Computer Science Department of the University of Potsdam, Germany, where he is working toward his Ph.D. degree. His research interest are the design of self-checking and self-testing circuits.

Prof. Valerij Saposhnikov received the M.S. degree (1963), the Ph.D. degree (1968) and the doctoral degree (1982) all in electrical engineering from the University of Railway Engineering, 
St. Petersburg (formerly Leningrad), Russia. In 1982 he became a professor, and in 1989 a vice president of this university. He published as an author and coauthor about 250 scientific papers and 8 books in russian language in the field of synthesis, diagnosis, self-checking circuits and control systems for railway transportation. Since 1992 he is a visiting professor of the Max-Planck Fault Tolerant Computing Group at the University of Potsdam, Germany. He is a member of the Russian Transportation Academy and of the International Academy of Higher Education.

Prof. Vladimir Saposhnikov received his M.S. degree (1963), the Ph.D. degree (1969) and the doctoral degree (1984) all in electrical engineering from the University of Railway Engineering St. Petersburg (formerly Leningrad), Russia. Since 1987 he is a professor and since 1991 the head of a department. Since 1992 he is a visiting professor of the Max-Planck Fault Tolerant Computing Group at the University of Potsdam, Germany. $\mathrm{He}$ is the author and coauthor of about 300 scientific publications and 8 books in Russian language. His research interests are synthesis and diagnosis of discrete systems, self-checking systems and fail-safe transportation systems. $\mathrm{He}$ is a member of the Russian Transportation Academy and of the International Academy of Higher Education.

Prof. Michael Goessel received the M.S. Degree (1965) and the Ph.D. Degree (1968), both in physics, from the University of Jena, Germany and the doctoral degree in Computer Science (1971) from the Technical University of Dresden, Germany. From 1966 to 1968 he was a research assistent at the University of Leningrad. From 1969 to 1991 he was with the Central Institute of Cybernetics (ZKI) of the Academy of Sciences, Berlin, of the formerly GDR. In 1992 he was appointed as the head of the Fault Tolerant Computing Group of the Max-Planck-Society at the University of Potsdam, Germany. Since 1994 he is a full-time professor of Computer Science at the University of Potsdam, Germany. He is the author and coauthor of 7 books, including "Error Detection Circuits", (Goessel, Graf, McGraw-Hill 1993) and "Memory Architecture and Parallel Access”, (Goessel, Rebel, Creutzburg, Wiley 1994). 

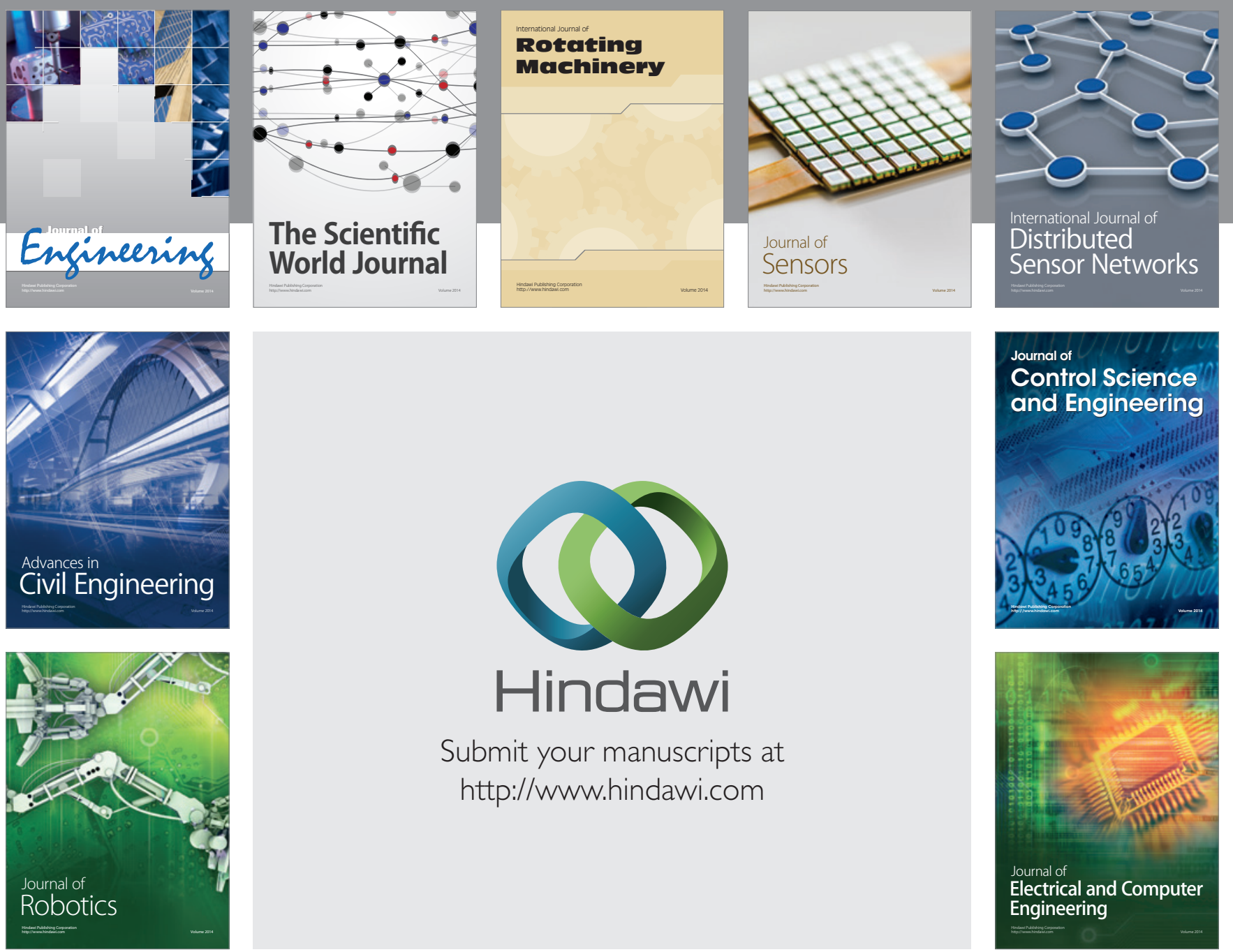

Submit your manuscripts at

http://www.hindawi.com
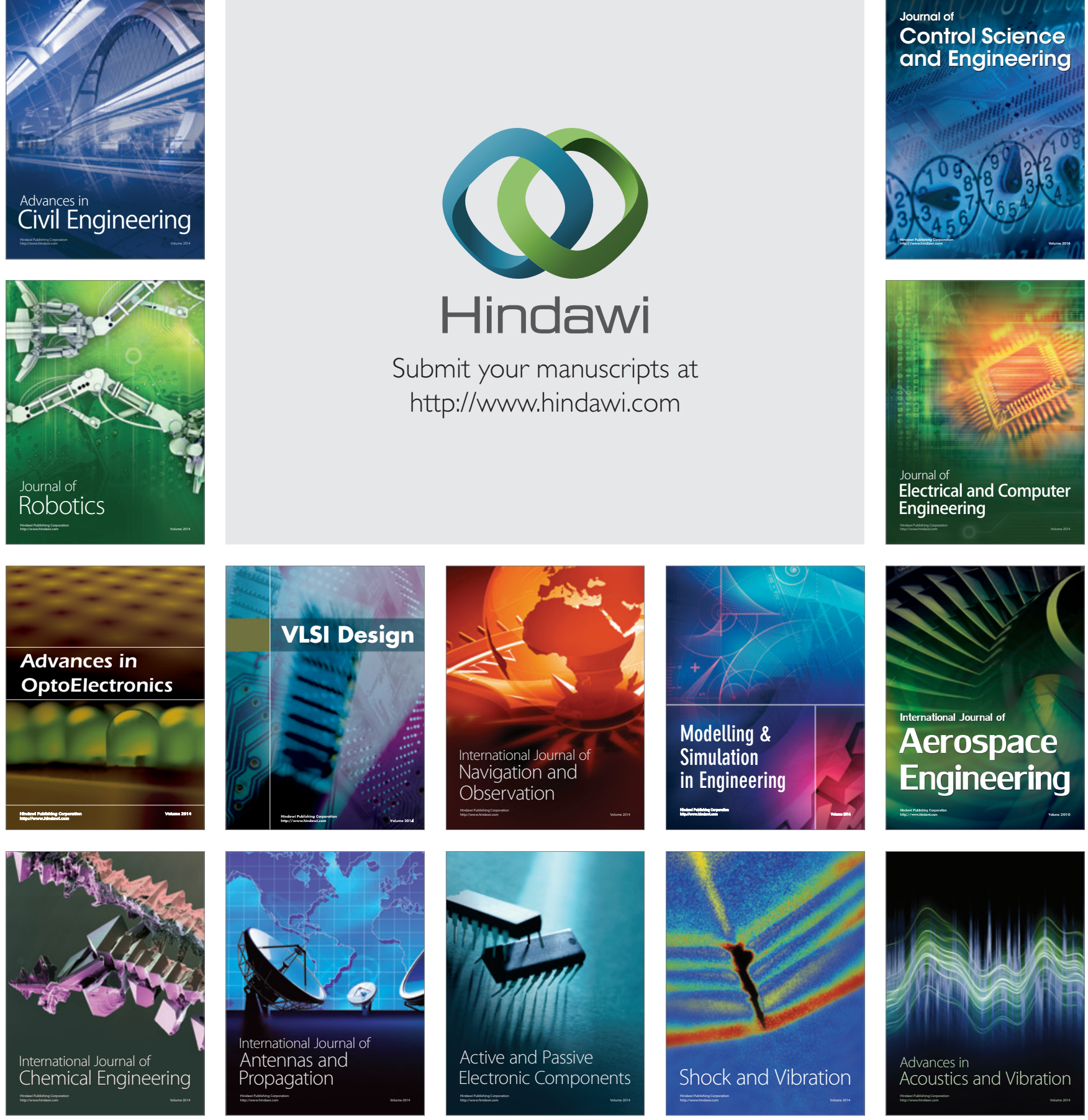